\title{
On the Properties of Runge-Kutta Discrete Adjoints
}

\author{
Adrian Sandu \\ Department of Computer Science, \\ Virginia Polytechnic Institute and State University, \\ Blacksburg, VA 24061 \\ sandu@cs.vt.edu
}

\begin{abstract}
In this paper we analyze the consistency and stability properties of Runge-Kutta discrete adjoints. Discrete adjoints are very popular in optimization and control since they can be constructed automatically by reverse mode automatic differentiation. The consistency analysis uses the concept of elementary differentials and reveals that the discrete Runge-Kutta adjoint method has the same order of accuracy as the original, forward method. A singular perturbation analysis reveals that discrete adjoints of stiff Runge-Kutta methods are well suited for stiff problems.
\end{abstract}

Keywords: Runge-Kutta methods, discrete adjoints.

\section{Introduction}

Consider the ordinary differential equation (ODE)

$$
y^{\prime}=f(t, y), \quad y\left(t_{0}\right)=y_{0}, \quad t_{0} \leq t \leq t_{\mathrm{F}} .
$$

We will denote the Jacobian of the ODE function by $J(t, y)=\partial f(t, y) / \partial y$.

We are interested in the following optimization problem, which arises in important applications like control and data assimilation. Find the initial conditions for which a function of the system state at the final time is minimized,

$$
\min _{y_{0}} \bar{\Psi}\left(y_{0}\right)=h\left(y\left(t_{\mathrm{F}}\right)\right) \quad \text { subject to (1) . }
$$

To apply a gradient based optimization procedure one needs to compute the derivatives of the cost function $\bar{\Psi}$ with respect to the initial conditions. It can be shown [6] that these derivatives can be obtained efficiently by solving the continuous adjoint equation

$$
\lambda^{\prime}=-J^{T}(t, y(t)) \lambda, \quad \lambda\left(t_{\mathrm{F}}\right)=\frac{\partial h}{\partial y}\left(y\left(t_{\mathrm{F}}\right)\right), \quad t_{\mathrm{F}} \geq t \geq t_{0}
$$

backwards in time from $t_{\mathrm{F}}$ to $t_{0}$ to obtain

$$
\lambda\left(t_{0}\right)=\frac{\partial \bar{\Psi}}{\partial y_{0}} .
$$


Note that the continuous adjoint equation (3) is formulated based on the forward solution $y(t)$.

In practice the equation (1) is solved numerically on a computer to obtain approximations of the ODE solution $y_{n} \approx y\left(t_{n}\right)$. Using a one-step numerical method (e.g., Runge-Kutta) the numerical solution is advanced in time as follows

$$
y_{n+1}=\mathcal{M}_{n}\left(y_{n}\right), \quad y_{N}=\mathcal{M}_{N-1}\left(\mathcal{M}_{N-2}\left(\cdots \mathcal{M}_{0}\left(y_{0}\right)\right)\right)
$$

where $t_{N}=t_{\mathrm{F}}$ and the numerical solution at the final time is $y_{N} \approx y\left(t_{\mathrm{F}}\right)$. The optimization problem (2) is formulated in terms of the numerical solution minimized,

$$
\min _{y_{0}} \Psi\left(y_{0}\right)=h\left(y_{N}\right) \quad \text { subject to (4) . }
$$

To estimate the gradient of the cost function (5) several approaches are possible. In the continuous adjoint approach one solves the continuous adjoint equation (3) backwards in time using any numerical discretization technique, e.g., a Runge-Kutta method. The terminal value of the adjoint variable $\lambda\left(t_{0}\right)$ is an approximation of the gradient of (2), and (hopefully) is also an approximation of the gradient of (5).

In the discrete adjoint approach the gradient of (2) is computed directly from (4) using the transposed chain rule

$$
\left(\frac{d \Psi}{d y_{0}}\right)^{T}=\left(\frac{d \mathcal{M}_{0}}{d y_{0}}\left(y_{0}\right)\right)^{T} \cdots\left(\frac{d \mathcal{M}_{N-1}}{d y_{N-1}}\left(y_{N-1}\right)\right)^{T}\left(\frac{d h}{d y_{N}}\left(y_{N}\right)\right)^{T} .
$$

This calculation proceeds backwards in time, i.e. the expression is evaluated right to left as follows

$$
\lambda_{N}=\left(\frac{d h}{d y_{N}}\left(y_{N}\right)\right)^{T} \ldots \quad \lambda_{n}=\left(\frac{d \mathcal{M}_{n}}{d y_{n}}\left(y_{n}\right)\right)^{T} \lambda_{n+1} \ldots \quad \lambda_{0}=\left(\frac{d \Psi}{d y_{0}}\right)^{T} .
$$

We will call $\lambda_{n}$ discrete adjoint variables. Their evaluation requires the forward numerical solution $y_{0}$ to $y_{N}$ to be available during the backward calculation.

Discrete adjoints are useful in optimization since they provide the gradients of the numerical function that is being minimized. Continuous adjoints are useful for sensitivity analysis studies. They are relatively easy to compute by applying a numerical solver of choice to the continuous equation (3), and using the forward solution $y(t)$ obtained by interpolation from a sequence of checkpoints.

The calculation of gradients by reverse automatic differentiation leads to the discrete adjoint approach. This paper is focused on analyzing some of the properties of the discrete adjoint variables $\lambda_{n}$ and their relationship with the continuous adjoint variables $\lambda\left(t_{n}\right)$ when the numerical integration (both forward and backward in time) is performed by Runge-Kutta methods.

Consistency properties of discrete Runge-Kutta adjoints have been studied by Hager [5], who gives additional order conditions necessary in the context of control problems. Walther [7] has studied the effects of reverse mode automatic 
differentiation on explicit Runge-Kutta methods, and finds that the order of the discretization is preserved by discrete adjoints. Giles [2] has discussed RungeKutta adjoints in the context of steady state flows. In this paper we consider control problems where only the initial conditions are the control variables. This setting is simpler than the distributed control case considered by Hager [5] and Walther [7.

\section{$1.1 \quad$ Runge-Kutta Methods}

A general $s$-stage Runge-Kutta discretization method is defined as [3, Section II.1]

$$
\begin{aligned}
y_{n+1} & =y_{n}+h \sum_{i=1}^{s} b_{i} k_{i}, \quad h=t_{n+1}-t_{n}, \\
k_{i} & =f\left(t_{n}+c_{i} h, Y_{i}\right), \quad Y_{i}=y_{n}+h \sum_{j=1}^{s} a_{i, j} k_{j},
\end{aligned}
$$

where the coefficients $a_{i, j}, b_{i}$ and $c_{i}$ are prescribed for the desired accuracy and stability properties. If $a_{i, j} \neq 0$ for $j \geq i$ the stage derivative values $k_{i}$ are defined implicitly, and are obtained by solving the nonlinear system (7).

Hager [5] has shown that the discrete adjoint (6) of the Runge-Kutta method (7) is

$$
\begin{aligned}
\lambda_{n} & =\lambda_{n+1}+\sum_{j=1}^{s} \theta_{j}, \\
\theta_{i} & =h J^{T}\left(t_{n}+c_{i} h, Y_{i}\right)\left(b_{i} \lambda_{n+1}+\sum_{j=1}^{s} a_{j, i} \theta_{j}\right), \quad i=1 \cdots s .
\end{aligned}
$$

Hager has also shown that if all $b_{i} \neq 0$ then the discrete adjoint reads

$$
\begin{aligned}
& \lambda_{n}=\lambda_{n+1}+h \sum_{i=1}^{s} \bar{b}_{i} \ell_{i}, \quad \ell_{i}=J^{T}\left(t_{n}+c_{i} h, Y_{i}\right) \Lambda_{i}, \\
& \Lambda_{i}=\lambda_{n+1}+h \sum_{j=1}^{s} \bar{a}_{i, j} \ell_{j}, \quad \text { with } \quad \bar{b}_{i}=b_{i}, \quad \bar{a}_{i, j}=\frac{a_{j, i} b_{j}}{b_{i}} .
\end{aligned}
$$

In the continuous adjoint approach one solves the equation (3) with a RungeKutta method (7) with coefficients $\tilde{a}_{i, j}, \tilde{b}_{i}, \tilde{c}_{i}$ to obtain

$$
\begin{aligned}
& \lambda_{n}=\lambda_{n+1}+h \sum_{i=1}^{s} \tilde{b}_{i} \tilde{\ell}_{i}, \\
& \tilde{\ell}_{i}=J^{T}\left(t_{n+1}-\tilde{c}_{i} h, y\left(t_{n+1}-\tilde{c}_{i} h\right)\right) \Lambda_{i}, \quad \Lambda_{i}=\lambda_{n+1}+h \sum_{j=1}^{s} \tilde{a}_{i, j} \tilde{\ell}_{j} .
\end{aligned}
$$




\section{Consistency of the Discrete Adjoint Method}

We now regard the discrete adjoint equation (8) as a numerical method applied to the continuous adjoint equation (3) and try to assess how accurate this numerical method is. At a first glance if $b_{i} \neq 0$ we can use the similarity between (9) and (10) and apply the standard Runge-Kutta order conditions to the method with coefficients $\bar{a}_{i, j}=\left(a_{j, i} b_{j}\right) / b_{i}, b_{i}, c_{i}$. The difficulty consists in the fact that in the continuous adjoint (10) the transposed Jacobian is evaluated using the exact numerical solution $y\left(t_{n+1}-\tilde{c}_{i} h\right)$, while in the discrete adjoint (9) the Jacobian is evaluated using the stage solutions in the forward method $Y_{i}$. Therefore the accuracy with which these stage solutions are evaluated in the forward run affects the accuracy of the discrete adjoint method.

In implicit Runge-Kutta processes $Y_{i}$ are the solutions of a nonlinear system of equations. The accuracy with which the nonlinear system is solved impacts further the accuracy of the discrete adjoint. In this paper we will analyze the order conditions under the assumption that the nonlinear systems are solved exactly in both the forward (7) and the discrete adjoint (8) methods. An additional complication is given by the fact that black-box application of automatic differentiation tools will result in a differentiation of the iterations needed to solve the nonlinear system in the forward method. A discussion of the behavior of the resulting (differentiated) iterations is beyond the scope of this paper.

Walther [7] found that the order of the discrete adjoints of explicit RungeKutta methods is the same as the order of the original method. In this section we will prove the same result in greater generality; our proof is applicable to both explicit and implicit Runge-Kutta methods.

Before we start the analysis we introduce the following "transfer functions". The discrete adjoint is obtained from the "discrete transfer function" $R_{D}$

$$
\lambda_{n}=\left(\frac{d y_{n+1}}{d y_{n}}\right)^{T} \lambda_{n+1}=R_{D} \lambda_{n+1}
$$

Similarly from the linear continuous adjoint equation (3) we derive a linear dependence between the continuous adjoint variables at different time, and call this dependence the "continuous transfer function" $R_{C}$ such that $\lambda\left(t_{n}\right)=$ $R_{C} \lambda\left(t_{n+1}\right)$.

The analysis of the order of discrete adjoints is based on the concept of elementary differentials in the theory of order conditions explained in Hairer et al. [3, section II.2]. The numerical solution of the forward Runge-Kutta method (7) satisfies

$$
\left.\left(y_{n+1}^{J}\right)^{(q)}\right|_{h=0}=\sum_{\tau \in L T_{q}} \gamma(\tau) \sum_{j} b_{j} \Phi_{j}(\tau) F^{J}(\tau)\left(y_{n}\right)
$$

where the superscipt $J$ denotes the component number, the first summation is taken after all labeled trees of order $q$, and $F(\tau)\left(y_{n}\right)$ is the elementary differential associated with $\tau . \Phi_{j}(\tau)$ is a combination of method coefficients associated with $\tau$ and $\gamma(\tau)$ is the multiplicity of tree $\tau$. 
The derivative of the solution $y_{n+1}$ with respect to $y_{n}$ satisfies

$$
\left.\left(\frac{\partial y_{n+1}^{J}}{\partial y_{n}^{P}}\right)^{(q)}\right|_{h=0}=\left.\frac{\partial}{\partial y_{n}^{P}}\left(y_{n+1}^{J}\right)^{(q)}\right|_{h=0}=\sum_{\tau \in L T_{q}} \gamma(\tau) \sum_{j} b_{j} \Phi_{j}(\tau) F_{P}^{J}(\tau)\left(y_{n}\right)
$$

where $F_{P}^{J}(\tau)\left(y_{n}\right)$ is the partial derivative of the elementary differential with respect to $P$-th argument, namely $y_{n}^{P}$. Consequently the $(P, J)$-th entry in the discrete transfer function has the following derivatives

$$
\left.\left(R_{D}^{P, J}\right)^{(q)}\right|_{h=0}=\sum_{\tau \in L T_{q}} \gamma(\tau) \sum_{j} b_{j} \Phi_{j}(\tau) F_{P}^{J}(\tau)\left(y_{n}\right)
$$

The exact solution of the direct system satisfies [3, Section II.2]

$$
\left.\left(y^{J}\right)^{(q)}\right|_{t=t_{n}}=\sum_{\tau \in L T_{q}} F^{J}(\tau)\left(y_{n}\right) .
$$

Consider now the continuous adjoint equation. With $\Delta t=t_{n}-t_{n+1}$ the Taylor series of $R_{C}$ about $t_{n+1}$ is

$$
\lambda\left(t_{n}\right)=R_{C} \lambda\left(t_{n+1}\right)=\sum_{j \geq 0} \frac{\Delta t^{q}}{q !} R_{C}^{(q)} \lambda\left(t_{n+1}\right)=\sum_{j \geq 0} \frac{\Delta t^{q}}{q !} \lambda^{(q)}\left(t_{n+1}\right)
$$

and therefore $R_{C}^{(q)}$ maps $\lambda\left(t_{n+1}\right)$ to $\lambda^{(q)}\left(t_{n+1}\right)$. It can be shown that the derivatives of the exact solution of the adjoint equation, taken with respect to $(-t)$ at $t_{n}$, are

$$
\left.\left(\lambda^{P}\right)^{(q)}\right|_{t=t_{n}}=\sum_{\tau \in L T_{q}} F_{P}^{J}(\tau)\left(y_{n}\right) \lambda^{J}\left(t_{n}\right) .
$$

This implies that for the continuous transfer function

$$
\left(R_{C}^{P, J}\right)^{(q)}=\sum_{\tau \in L T_{q}} F_{P}^{J}(\tau)\left(y_{n}\right) .
$$

For example taking the derivative w.r.t. $(-t)$ in (3) gives

$$
\lambda^{(1)}=J^{T}(t, y(t)) \lambda \Rightarrow\left(\lambda^{P}\right)^{(1)}=\sum_{J} f_{P}^{J} \lambda^{J}=\sum_{J} F_{P}^{J}\left(\tau_{1}\right) \lambda^{J} .
$$

This implies that for the continuous transfer function

$$
\left(R_{C}^{P, J}\right)^{(1)}=F_{P}^{J}\left(\tau_{1}\right)\left(y_{n}\right) .
$$

Similarly

$$
\begin{aligned}
\left(\lambda^{P}\right)^{(2)} & =\sum_{J, K} f_{P K}^{J} f^{K} \lambda^{J}+\sum_{J} f_{P}^{J}\left(\lambda^{J}\right)^{(1)}=\sum_{J, K} f_{P K}^{J} f^{K} \lambda^{J}+\sum_{J, K} f_{P}^{J} f_{J}^{K} \lambda^{K} \\
& =\sum_{J, K}\left(f_{P K}^{J} f^{K}+\sum_{J, K} f_{K}^{J} f_{P}^{K}\right) \lambda^{J}=\sum_{J} F_{P}^{J}\left(\tau_{21}\right) \lambda^{J}
\end{aligned}
$$


and therefore for the continuous transfer function we have

$$
\left(R_{C}^{P, J}\right)^{(2)}=F_{P}^{J}\left(\tau_{21}\right)\left(y_{n}\right)
$$

Continuing this process one obtains (16).

A comparison of (13) and (16) shows that the numeric transfer function equals the continuous transfer function up to order $p$ iff

$$
\sum_{j} b_{j} \Phi_{j}(\tau)=\frac{1}{\gamma(\tau)}
$$

for all trees $\tau$ of order $\leq p$. But these are exactly the conditions under which the forward method is of order $p$. Consequently, the discrete adjoint of an order $p$ Runge-Kutta method is a discretization of order $p$ of the continuous adjoint equation if the problem is sufficiently smooth $(y(t)$ has $\mathrm{p}+1$ continuous derivatives).

\section{Discrete Adjoints and Stiff Problems}

The traditional linear stability analysis approach [4] considers the transfer function $R(z)$ of a Runge-Kutta method when applied to a linear scalar test problem $y^{\prime}=\alpha y$. One can easily see that the transfer function of the discrete adjoint method (8) is the same as the transfer function of the forward Runge-Kutta method (7), and therefore the discrete adjoints inherits the stability properties of the original method.

To better understand the behavior of discrete Runge-Kutta adjoints on stiff systems we consider the singular perturbation model problem

$$
y^{\prime}=f(y, z), \quad \epsilon z^{\prime}=g(y, z), \quad t_{0} \leq t \leq t_{F}
$$

with the sub-Jacobian $g_{z}$ assumed nonsingular. For this system we distinguish between the adjoint variables of the nonstiff and of the stiff components

$$
\lambda(t)=\frac{\partial \Psi\left(y\left(t_{\mathrm{F}}\right), z\left(t_{\mathrm{F}}\right)\right)}{\partial y(t)}, \quad \mu(t)=\frac{\partial \Psi\left(y\left(t_{\mathrm{F}}\right), z\left(t_{\mathrm{F}}\right)\right)}{\partial z(t)} .
$$

The adjoint variables satisfy the continuous adjoint equation

$$
\left[\begin{array}{l}
\lambda \\
\mu
\end{array}\right]^{\prime}=-\left[\begin{array}{cc}
f_{y}^{T} & \epsilon^{-1} g_{y}^{T} \\
f_{z}^{T} & \epsilon^{-1} g_{z}^{T}
\end{array}\right]\left[\begin{array}{l}
\lambda \\
\mu
\end{array}\right]
$$

Consider an $\epsilon$-expansion of the solution

$$
\lambda=\sum_{i \geq 0} \epsilon^{i} \lambda^{i}, \quad \mu=\sum_{i \geq 0} \epsilon^{i} \mu^{i}
$$


insert it into (18) and equate the $\epsilon$ series. The $\epsilon^{-1}$ term leads to

$$
\mu^{0}=0
$$

We equate recursively the higher order terms to obtain

$$
\left\{\begin{array}{l}
\left(\lambda^{i}\right)^{\prime}=\left(-f_{y}^{T}+g_{y}^{T} g_{z}^{-T} f_{z}^{T}\right) \lambda^{i}+g_{y}^{T} g_{z}^{-T}\left(\mu^{i}\right)^{\prime} \\
\mu^{i+1}=-g_{z}^{-T}\left(\left(\mu^{i}\right)^{\prime}+f_{z}^{T} \lambda^{i}\right),
\end{array} \quad \text { for } \quad i=0,1,2, \ldots\right.
$$

The zeroth order term evolution is given by the equation

$$
\left(\lambda^{0}\right)^{\prime}=\left(-f_{y}^{T}+g_{y}^{T} g_{z}^{-T} f_{z}^{T}\right) \lambda^{0} \text {. }
$$

Consider now the Runge-Kutta discrete adjoint (8) for the method applied to the singular perturbation systems (17). Denote by $A, b, c$ the coefficient matrices of the Runge-Kutta method, by $e$ a vector of ones, and let

$$
\begin{aligned}
G_{Z} & =\operatorname{diag}_{i}\left(g_{z}\left(Y_{i}, Z_{i}\right)\right), \quad F_{Z}=\operatorname{diag}_{i}\left(f_{z}\left(Y_{i}, Z_{i}\right)\right), \\
G_{Y} & =\operatorname{diag}_{i}\left(g_{y}\left(Y_{i}, Z_{i}\right)\right), \quad F_{Y}=\operatorname{diag}_{i}\left(f_{y}\left(Y_{i}, Z_{i}\right)\right), \\
S^{T} & =F_{Y}^{T}-G_{Y}^{T} G_{Z}^{-T} F_{Z}^{T},
\end{aligned}
$$

A careful analysis based on $\epsilon$-expansions of the discrete solution and of the stage vectors leads to the following conclusions.

If the Runge-Kutta coefficient matrix $A$ is invertible then the adjoints of the stiff variables are integrated using

$$
\mu_{n}^{0}=R(\infty) \mu_{n+1}^{0},
$$

where $R(\cdot)$ is the stability function of the RK method. We see easily that for methods with $R(\infty)=0$ the first order term in the $\mu$ adjoint is zero, $\mu_{n}^{0}=0$. This is desirable considering the exact solution (20). In this case the values of $\mu$ are solved with the same accuracy as the original method, within $\mathcal{O}(\epsilon)$.

The adjoints of the non-stiff variables are integrated as

$$
\begin{aligned}
\lambda_{n}^{0}= & \left(1+h e^{T}\left(I-h S^{T} A^{T}\right)^{-1} S^{T} b\right) \lambda_{n+1}^{0} \\
& +e^{T}\left(I-h S^{T} A^{T}\right)^{-1} G_{Y}^{T} G_{Z}^{-T} A^{-T} b \mu_{n+1}^{0}
\end{aligned}
$$

Therefore the adjoint with respect to the nonstiff variable depends on both $\mu_{n+1}^{0}$ and $\lambda_{n+1}^{0}$.

If $R(\infty)=0$ and we are away from the initial condition then $\mu_{n+1}^{0}=0$ and we have a discretization of the reduced adjoint equation (21). The same holds if the cost function depends only on the non-stiff variables, $\Psi=\Psi(y)$. In this case the values of $\lambda$ are solved with the same accuracy as the original method, within $\mathcal{O}(\epsilon)$. Note that the initialization of $\mu_{N}^{0} \neq 0$ can introduce an $\mathcal{O}(1)$ perturbation in $\lambda_{N-1}^{0}$. 


\section{Conclusions}

In this paper we analyze the consistency and stability properties of Runge-Kutta discrete adjoints. Discrete adjoints are very popular in optimization and control since they can be constructed automatically by reverse mode automatic differentiation. However, the properties of the discrete adjoints are often poorly understood.

The consistency analysis uses the concept of elementary differentials and reveals that the Runge-Kutta discrete adjoint method has the same order of accuracy as the original, forward method. The discrete adjoint also inherits the linear stability properties of the original method. A singular perturbation analysis shows that L-stable Runge-Kutta methods with an invertible coefficient matrix are well-behaved under discrete adjoint operation.

\section{Acknowledgments}

This work was supported by the National Science Foundation (NSF) through the awards NSF CAREER ACI-0413872, NSF ITR AP\&IM 020519, and NSF CCF0515170, by the National Oceanic and Atmospheric Administration (NOAA) and by the Texas Environmental Research Consortium (TERC).

\section{References}

1. M.L. Baguer and W. Romisch. Computing gradients in parametrizationdiscretization schemes for constrained optimal control problems. Approximation and Optimization in the Carribbean II, p. 14-34, M. Florenzano editor, Peter Lang, Frankfurt am Main, 1995.

2. M.B. Giles. On the Use of Runge-Kutta Time-Marching and Multigrid for the Solution of Steady Adjoint Equations. Technical Report NA00/10, Oxford University Computing Laboratory, 2000.

3. E. Hairer, G. Wanner, and S.P. Norsett. Solving Ordinary Differential Equations I. Nonstiff Problems. Springer Series in Computational Mathematics, 1991.

4. E. Hairer and G. Wanner. Solving Ordinary Differential Equations II. Stiff and Differential-Algebraic Problems. Springer Series in Computational Mathematics, 1996.

5. W. Hager. Runge-Kutta methods in optimal control and the transformed adjoint system. Numerische Mathematik 87(2):247-282, 2000.

6. A. Sandu, D. Daescu, and G.R. Carmichael: "Direct and Adjoint Sensitivity Analysis of Chemical Kinetic Systems with KPP: I - Theory and Software Tools", Atmospheric Environment, Vol. 37, p. 5083-5096, 2003.

7. A Walther. Automatic Differentiation of Explicit Runge-Kutta methods for Optimal Control. Technical University Dresden technical report WR-06-2004. To appear in Journal of Computational Optimization and Applications. 